\title{
Fully differential NLO predictions for rare and radiative lepton decays
}

\author{
Y. Ulrich* \\ Paul Scherrer Institut, \\ CH-5232 Villigen PSI, Switzerland \\ Physik-Institut, Universität Zürich, \\ Winterthurerstrasse 190, \\ CH-8057 Zürich, Switzerland \\ E-mail: yannick.ulrich@psi.ch
}

\begin{abstract}
We present a general purpose, parton-level Monte Carlo program for the calculation of the radiative $(L \rightarrow v \bar{v} l+\gamma)$ and rare $\left(L \rightarrow v \bar{v} l^{\prime}+l^{+} l^{-}\right)$muon and tau decays at NLO in the effective Fermi theory with $L \in\{\tau, \mu\}$ and $l, l^{\prime} \in\{\mu, e\}$. In the case of muon, these processes are irreducible Standard Model backgrounds to searches for lepton flavour violation at the PSI experiments MEG and $\mathrm{Mu} 3 \mathrm{e}$ as they become indistinguishable from the corresponding signals when the neutrinos carry little energy.

Furthermore, we argue that fully differential NLO corrections are very important for the analysis of measurements aiming at the percent level or better. This is especally true if very stringent phase-space cuts are applied. To illustrate this, we use a tension between BABAR's recent measurement of the radiative tau decay and the Standard Model prediction as an example of such an analysis. Finally, we present the branching ratios of the rare tau decay $\tau \rightarrow v \bar{v} l^{\prime} l^{+} l^{-}$at NLO. We generally find that QED corrections of $\mathscr{O}(10 \%)$ are very well possible.
\end{abstract}

The 19th International Workshop on Neutrinos from Accelerators-NUFACT2017

25-30 September, 2017

Uppsala University, Uppsala, Sweden

*Speaker. 


\section{Introduction}

Muon decays have a long and rich history in particle physics and will no doubt continue to play an important rule. Their study, both experimentally and theoretically, helped to precisely determine standard model quantities like the Fermi constant $G_{F}$ and severely constrain lepton flavour violating (LFV) new physics [1]. While radiative corrections to the Michel decay are available for many decades, NLO results for the radiative $\mu \rightarrow v \bar{v} e \gamma$ and rare $\mu \rightarrow v \bar{v} e e^{+} e^{-}$decay have only been published in the last two years $[2,3,4,5]$.

In the case of $\tau$ decays, precision measurements at the percent level are available. Especially for the radiative $\tau$ decay there has been a discrepancy at the level of several $\sigma$.

Here we will review the rare and radiative lepton decays in their role as SM background processes to the searches for LFV by the experiments MEG [6] and Mu3e [7, 8] and further stress the importance of fully differential calculations.

The framework of our calculation is the QED Lagrangian augmented with the Fierz rearranged effective Fermi interaction

$$
\mathscr{L}=\mathscr{L}_{\mathrm{QED}}+\frac{4 G_{F}}{\sqrt{2}}\left(\bar{\psi}_{e} \gamma^{\mu} P_{L} \psi_{\mu}\right)\left(\bar{\psi}_{v_{\mu}} \gamma^{\mu} P_{L} \psi_{v_{e}}\right)+\text { h.c. }
$$

where $P_{L}=\left(1-\gamma_{5}\right) / 2$ is the usual left-handed projector. The QED part contains the necessary lepton fields, i.e. $\psi_{\mu}$ and $\psi_{e}$. Even though this is an effective field-theoretical description of the underlying electroweak interaction, it has been shown by Berman and Sirlin [9] that QED corrections are finite at all orders and that $G_{F}$ does not need to be renormalised. Unless noted otherwise, we keep the electron mass $m_{e} \neq 0$.

We compute the one-loop diagrams with a modified version of GoSam $[10,11,12]$ as well as FeynArts [13], FormCalc [14] and LoopTools [15]. Infrared singularities in the real corrections are dealt with using FKS subtraction [16, 17]. Finally, we integrate the phase space using VEGAS [18]. This allows us to create arbitrary distributions involving arbitrary cuts. Further details regarding the implementation are available in $[4,5]$.

\section{Radiative tau decay $\tau \rightarrow v \bar{v} e \gamma$}

Aside from being an important background to searches for $\mu \rightarrow e \gamma$, the radiative lepton decays $L \rightarrow v \bar{v} l+\gamma$ can be used as an example to illustrate why fully differential Monte Carlo programs are important. This can be seen best in the radiative tau decay $\tau \rightarrow v \bar{v} e+\gamma$ which was measured by BABAR [19]. As observed by Fael, Mercolli and Passera [2], the measured value and the NLO branching ratio disagree at around $3.5 \sigma$.

Experimentally, the following happens: a pair of tau leptons is created in $e^{+} e^{-}$collisions at $\sqrt{s}=M_{\Upsilon(4 S)}=10.58 \mathrm{GeV}$. One of the taus is used for tagging, while the signal is measured with the other tau. Very stringent cuts are imposed on the signal tau in the centre-of-mass frame of the $e^{+} e^{-}$pair to reduce background events

$$
\cos \theta_{e \gamma}^{*} \geq 0.97, \quad 0.22 \mathrm{GeV} \leq E_{\gamma}^{*} \leq 2.0 \mathrm{GeV}, \quad M_{e \gamma} \geq 0.14 \mathrm{GeV} .
$$


By implementing these cuts in our Monte Carlo program, we will see, that the NLO corrections will have an important effect when 'undoing' the cuts, i.e. when extracting exclusive branching ratio $^{1} \mathscr{B}^{\text {excl }}$ (with only the cut $E_{\gamma} \geq 10 \mathrm{MeV}$ in the tau rest frame).

To account for this effect we use the following simplified scheme: assume that $N_{\text {obs }}$ events passed the cuts (2.1). To transform $N_{\mathrm{obs}}$ into a branching fraction, it is multiplied by a factor $\varepsilon_{\mathrm{LO}}^{\text {exp }}$

$$
\mathscr{B}_{\exp }^{\mathrm{LO}}=\varepsilon_{\mathrm{LO}}^{\mathrm{exp}} \cdot N_{\mathrm{obs}}=\varepsilon_{\mathrm{det}} \cdot \varepsilon_{\mathrm{LO}} \cdot N_{\mathrm{obs}}=1.834(1) \cdot 10^{-2} .
$$

Detector effects are assumed to be described by $\varepsilon_{\text {det }}$ while $\varepsilon_{\mathrm{LO}}$ is a purely theoretical factor that converts the fiducial branching ratio to the desired value. The computation of this factor can be done both at LO and NLO

$$
\varepsilon_{\mathrm{LO}}=\left.\frac{\Gamma_{\mathrm{LO}}^{10 \mathrm{MeV}}}{\Gamma_{\mathrm{LO}}^{\text {fiducial }}}\right|_{\text {theory }}=48.55(1), \quad \varepsilon_{\mathrm{NLO}}=\left.\frac{\Gamma_{\mathrm{NLO}}^{10 \mathrm{MeV}}}{\Gamma_{\mathrm{NLO}}^{\text {fiducial }}}\right|_{\text {theory }}=44.80(1),
$$

where $\Gamma^{10 \mathrm{MeV}}\left(\Gamma^{\mathrm{fiducial}}\right)$ refers to the $10 \mathrm{MeV}$ (fiducial) cut. We can calculate the value of $\mathscr{B}_{\text {exp }}$ applying an NLO Monte Carlo

$$
\mathscr{B}_{\exp }^{\mathrm{NLO}}=\frac{\varepsilon_{\mathrm{NLO}}}{\varepsilon_{\mathrm{LO}}} \cdot \mathscr{B}_{\exp }^{\mathrm{LO}}=\varepsilon^{\prime} \cdot \mathscr{B}_{\exp }^{\mathrm{LO}}=1.704(50) \cdot 10^{-2},
$$

with $\varepsilon^{\prime}=0.923(1)$. This reduces the discrepancy from $3.5 \sigma$ to $1.2 \sigma$.

Obviously, this is only a very simplistic and by far not complete simulation of the full analysis. Hence, we do not claim that this is the conclusive resolution of the discrepancy. However, we do point out that even in QED a proper inclusion of NLO effects is mandatory for a precision around the percent level. Further details of this analysis can be found in [5]. In light of recent $B$-anomalies it is worth noting, that QED corrections can be large and should be included whenever possible.

\section{Radiative muon decay $\mu \rightarrow v \bar{v} e \gamma$}

Let us first look at the PiBeta experiment [20], which used

$$
E_{\gamma} \geq 10 \mathrm{MeV} \text { and } \varangle\left(\vec{p}_{\gamma}, \vec{p}_{e}\right)>30^{\circ} .
$$

While the PiBeta measurement offers sub-percent precision, it was noted before [5, 21] that there is a significant disagreement between the quoted value for the standard model and our calculation. However, we can reproduce the quoted standard model value if we redo our calculation with vanishing electron mass $m_{e}=0$ and the inclusion of a factor to account for conversion into an $e^{+} e^{-}$pair [22]. Using the approach explained above, we can convert the PiBeta measurement of $\mathscr{B}_{\pi \beta}=4.365(42) \cdot 10^{-3}$ into

$$
\mathscr{B}_{\pi \beta}^{*}=\varepsilon_{\pi \beta}^{\prime} \mathscr{B}_{\pi \beta}=4.18(4) \cdot 10^{-3}
$$

which is compatible with the theoretical prediction of $\mathscr{B}=\left(4.26-0.04_{\mathrm{NLO}}\right) \cdot 10^{-3}$.

We can use these considerations to compare and contrast all major measurements of the radiative muon decay that are currently listed in the PDG. This is shown in Table 3.

\footnotetext{
${ }^{1}$ We always normalise the branching ration to the measured life time and not its LO order result
} 


\begin{tabular}{|c|c|c|c|}
\hline Experiment & $\varepsilon$ & $\mathscr{B}_{\text {meas. }}$ & $10^{2} \cdot \mathcal{E} \cdot \mathscr{B}_{\text {meas }}$ \\
\hline MEG [6] & $2.2 \cdot 10^{5}$ & $6.0(5) \cdot 10^{-8}$ & $1.3(1)$ \\
\hline PiBeta [20] & 2.9 & $4.18(4) \cdot 10^{-3}$ & $1.27(1)$ \\
\hline [23] & 1.0 & $1.4(4) \cdot 10^{-2}$ & $1.4(4)$ \\
\hline Average & & & $1.27(1)$ \\
\hline
\end{tabular}

Table 1: Various measurements of the radiative muon decay related the canonical configuration with $E_{\gamma} \geq$ $10 \mathrm{MeV}$ using the kinematic acceptance $\varepsilon=\mathscr{B}^{\text {th }}(10 \mathrm{MeV}) / \mathscr{B}^{\text {th }}$ (fiducial)

For MEG, which uses $85 \%$ polarised muons, we define the $\vec{z}$ axis along the muon polarisation, i.e. $\vec{P}_{\mu}=-0.85 \vec{z}$. We now can model the cuts used by the MEG experiment as

$$
\begin{aligned}
E_{\gamma}>40 \mathrm{MeV}, & E_{e}>45 \mathrm{MeV}, \\
\left|\cos \varangle\left(\vec{p}_{\gamma}, \vec{z}\right)\right| \equiv\left|\cos \theta_{\gamma}\right|<0.35, & \left|\phi_{\gamma}\right|>\frac{2 \pi}{3}, \\
\left|\cos \varangle\left(\vec{p}_{e}, \vec{z}\right)\right| \equiv\left|\cos \theta_{e}\right|<0.5, & \left|\phi_{e}\right|<\frac{\pi}{3} .
\end{aligned}
$$

Further, we require that exactly one photon with an energy larger than the detector threshold of roughly $2 \mathrm{MeV}$ hits the detector. Therefore, we reject a second photon that hits the detector if its energy is larger than $2 \mathrm{MeV}$, i.e.

$$
E_{\gamma_{2}}<\left\{\begin{array}{ll}
2 \mathrm{MeV} & \text { if }(3.1 \mathrm{~b}) \text { is satisfied } \\
\infty & \text { otherwise }
\end{array} .\right.
$$

This allows us to calculate for example the distribution w.r.t. the invisible energy $\not \subset=m_{\mu}-E_{e}-E_{\gamma}$ as shown in Figure 2. The corrections are fairly large $\mathscr{O}(5-10 \%)$ but always negative. This means that a background study that only used a LO calculation will actually produce a more conservative approximation.

\section{Rare muon decay $\mu \rightarrow v \bar{v} e e^{+} e^{-}$}

For the rare decay we use

$$
E_{e^{ \pm}}>10 \mathrm{MeV} \quad \text { and } \quad\left|\cos \varangle\left(\vec{p}_{e^{ \pm}}, \vec{e}_{z}\right)\right|<0.8
$$

to roughly approximate the Mu3e detector [8]. We can again create the invisible energy distribution as shown in Figure 3. The corrections are again very large for QED but still negative in the region of any potential signal.

While the observation of $\mu \rightarrow$ eee would be a clear indication of physics beyond the standard model, we would like to propose a different way to use a precise measurement of the rare muon decay to search for light new mediators: the Lorentz structure of a new mediator could be encoded in angular distributions of the $e^{+} e^{-}$pair. This is especially promising because theoretical uncertainties on these observables are very small as they only receive small corrections at the percent level. Furthermore, these corrections are very flat and barely distort the distribution's shape. Therefore, we can expect that the NNLO corrections to the shape would be well below the per-mille level. 


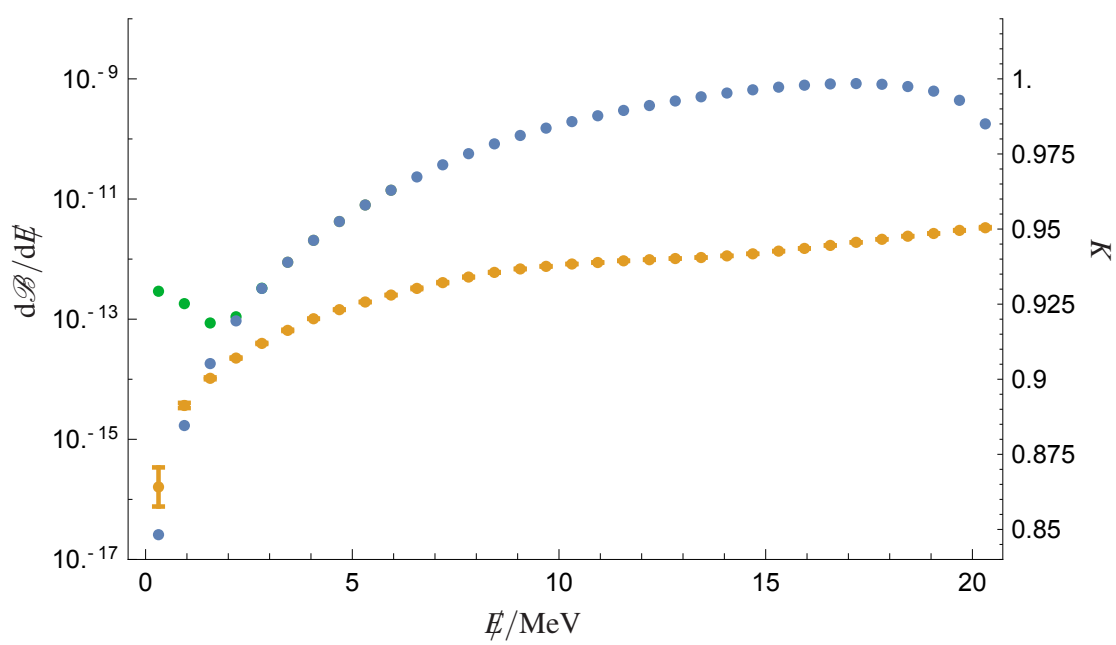

Figure 2: The invisible energy spectrum for MEG in blue at NLO, the $K$-factor as the ratio between NLO and LO in orange and in green a mock-up of how LFV would look like at the current limit $\mathscr{B}^{\mathrm{NP}} \simeq 4.2 \cdot 10^{-13}$ accounting for a $1.7 \%$ energy resolution.

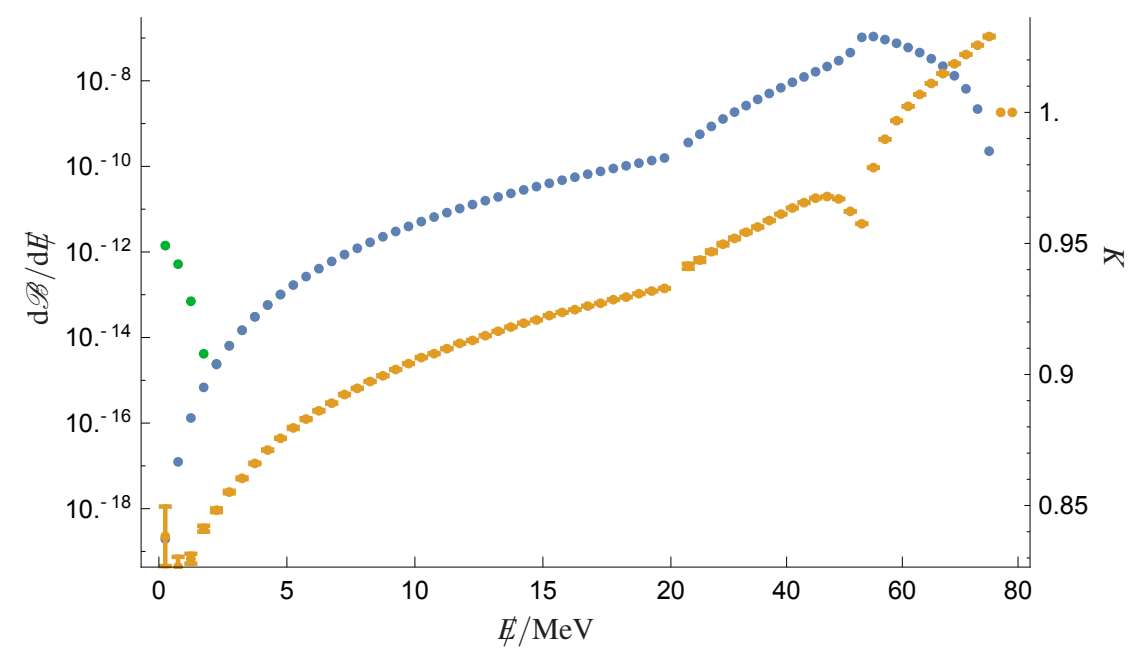

Figure 3: The invisible energy spectrum for Mu3e in blue at NLO, the $K$-factor as the ratio between NLO and LO in orange and in green a mock-up of how LFV would look like at the current limit $\mathscr{B}^{\mathrm{NP}} \simeq 10^{-12}$ accounting for a $0.5 \mathrm{MeV}$ energy resolution. 


\section{Rare tau decay $\tau \rightarrow v \bar{v} l^{\prime} l^{+} l^{-}$}

The rare tau decay $\tau \rightarrow v \bar{v} l^{\prime} l l$ has a richer phenomenology than the muon decay because more decay channels are possible. In fact, a plethora of tree-level calculations have been published using a variety of techniques $[24,25,26]$. On the other hand, experimental data is scarce. Nevertheless, we have used our program to calculate all four rare leptonic tau decay channels at NLO. Our results are summarised in Table 4 and compared with [25]. While our result includes all mass-effects it is not clear how reference [25] treated the light lepton's mass which cannot be set to zero because that would give rise to IR singularities. When computing the radiative corrections we have all three lepton-flavours active but neglect hadronic corrections because a rough estimate shows that they do not change the overall picture [4].

\begin{tabular}{c|c|c|c} 
& \multicolumn{2}{|c|}{ This work } & [25] \\
& LO & $\delta \mathscr{B} / \mathscr{B}$ & \\
\hline $10^{5} \mathscr{B}(\tau \rightarrow v \bar{v} e e e)$ & $4.2489(1)$ & $-0.094 \%$ & $4.22(2)$ \\
$10^{7} \mathscr{B}(\tau \rightarrow v \bar{v} \mu e e)$ & $1.9879(2)$ & $1.1 \%$ & $1.987(3)$ \\
$10^{5} \mathscr{B}(\tau \rightarrow v \bar{v} \mu \mu e)$ & $1.2513(2)$ & $1.2 \%$ & $1.246(2)$ \\
$10^{7} \mathscr{B}(\tau \rightarrow v \bar{v} \mu \mu \mu)$ & $1.1838(1)$ & $1.9 \%$ & $1.184(1)$
\end{tabular}

Table 4: The branching ratio of the rare tau decays computed at LO (by us and [25]) and NLO. We neglect hadronic corrections.

\section{Conclusion}

We have reviewed the NLO QED predictions for radiative $L \rightarrow l v \bar{v}+\gamma$ and rare $L \rightarrow l v \bar{v}+$ $l^{+} l^{-}$lepton decays with $L \in\{\tau, \mu\}$ and $l, l^{\prime} \in\{\mu, e\}$. In particular, we emphasise the importance of radiative corrections in unfolding fiducial acceptance when comparing experimental values with the PDG.

Furthermore, our program is able to calculate arbitrary differential distributions. For the experiments MEG and Mu3e, the corrections to these distributions is usually at the percent level. But especially in the critical regions of phase space they can easily reach $\mathscr{O}(10 \%)$.

\section{Acknowledgments}

The author is grateful to Matteo Fael and Massimo Passera for comparing and discussing our calculations. Furthermore, we are indebted to Angela Papa, Giada Rutar, Ann-Kathrin Perrevoort, Alberto Lusiani and Dinko Pocanic for their explaination of the experimental details underlying MEG, Mu3e, BABAR and Pibeta. The author is supported by he Swiss National Science Foundation (SNF) under contract 200021_163466.

\section{References}

[1] Y. Kuno and Y. Okada, Muon decay and physics beyond the standard model, Rev. Mod. Phys. 73 (2001) 151-202, [hep-ph/9909265]. 
[2] M. Fael, L. Mercolli and M. Passera, Radiative $\mu$ and $\tau$ leptonic decays at NLO, JHEP 07 (2015) 153, [1506.03416].

[3] M. Fael and C. Greub, Next-to-leading order prediction for the decay $\mu \rightarrow e\left(e^{+} e^{-}\right) v \bar{v}, J H E P(01$ (2017) 084, [1611.03726].

[4] G. M. Pruna, A. Signer and Y. Ulrich, Fully differential NLO predictions for the rare muon decay, Phys. Lett. B765 (2017) 280-284, [1611.03617].

[5] G. M. Pruna, A. Signer and Y. Ulrich, Fully differential NLO predictions for the radiative decay of muons and taus, Phys. Lett. B772 (2017) 452-458, [1705. 03782].

[6] MEG collaboration, A. M. Baldini et al., Measurement of the radiative decay of polarized muons in the MEG experiment, Eur. Phys. J. C76 (2016) 108, [1312 . 3217].

[7] Mu3E collaboration, N. Berger, The Mu3e Experiment, Nucl.Phys.Proc.Suppl. 248-250 (2014) 35-40.

[8] MU3E collaboration, A.-K. Perrevoort, Status of the Mu3e Experiment at PSI, EPJ Web Conf. 118 (2016) 01028, [1605.02906].

[9] S. Berman and A. Sirlin, Some considerations on the radiative corrections to muon and neutron decay, Annals of Physics 20 (1962) $20-43$.

[10] G. Cullen et al., GOSAM-2.0: a tool for automated one-loop calculations within the Standard Model and beyond, Eur. Phys. J. C74 (2014) 3001, [1404 . 7096].

[11] P. Mastrolia, E. Mirabella and T. Peraro, Integrand reduction of one-loop scattering amplitudes through Laurent series expansion, JHEP 06 (2012) 095, [1203 . 02 91].

[12] T. Peraro, Ninja: Automated Integrand Reduction via Laurent Expansion for One-Loop Amplitudes, Comput. Phys. Commun. 185 (2014) 2771-2797, [1 403 . 1229].

[13] T. Hahn, Generating Feynman diagrams and amplitudes with FeynArts 3, Comput.Phys.Commun. 140 (2001) 418-431, [hep-ph/0012260].

[14] B. Chokoufe Nejad, T. Hahn, J.-N. Lang and E. Mirabella, FormCalc 8: Better Algebra and Vectorization, J.Phys.Conf.Ser. 523 (2014) 012050, [1310.0274].

[15] T. Hahn and M. Perez-Victoria, Automatized one loop calculations in four-dimensions and D-dimensions, Comput.Phys.Commun. 118 (1999) 153-165, [hep-ph/9807565].

[16] S. Frixione, Z. Kunszt and A. Signer, Three-jet cross sections to next-to-leading order, Nuclear Physics B 467 (May, 1996) 399-442, [hep-ph/9512328v1].

[17] R. Frederix, S. Frixione, F. Maltoni and T. Stelzer, Automation of next-to-leading order computations in QCD: the FKS subtraction, Journal of High Energy Physics 2009 (Oct, 2009), [0908 . 4272v2].

[18] G. P. Lepage, VEGAS: An adaptive multidimensional integration program, .

[19] B. Oberhof, Measurement of $\mathscr{B}(\tau \rightarrow l \gamma v \bar{v}, l=e, \mu)$ at BaBar, Ph.D. thesis, University of Pisa, Italy, 2015.

[20] D. Pocanic et al., New results in rare allowed muon and pion decays, Int. J. Mod. Phys. Conf. Ser. 35 (2014) 1460437, [1403.7416].

[21] M. Fael and M. Passera, private communication, .

[22] S. Eckstein and R. Pratt, Radiative muon decay, Annals of Physics 8 (1959) 297 - 309. 
[23] R. R. Crittenden, W. D. Walker and J. Ballam, Radiative decay modes of the muon, Phys. Rev. 121 (1961) 1823-1832.

[24] A. Flores-Tlalpa, G. López Castro and P. Roig, Five-body leptonic decays of muon and tau leptons, JHEP 04 (2016) 185, [1508 . 01822$].$

[25] M. A. Arroyo-Ureña, E. Díaz, O. Meza-Aldama and G. Tavares-Velasco, $\tau^{-} \rightarrow \ell_{i}^{-} \ell_{i}^{+} \ell_{j}^{-} \bar{v}_{j} v_{\tau}$ decays with a magnetic dipole term, Int. J. Mod. Phys. A32 (2017) 1750195, [1711. 01393].

[26] D. A. Dicus and R. Vega, Standard Model decays of tau into three charged leptons, Phys. Lett. B338 (1994) 341-348, [hep-ph/9402262]. 